\title{
Where do the leukaemia relapses come from?
}

\author{
Arndt Borkhardt
}

Published online: 6 February 2010

(C) Springer-Verlag 2010

Since the first report of detecting minimal residual tumour cells during and after chemotherapy by a-at that time really sophisticated-technique named PCR 23 years ago [1], the approach has significantly changed our clinical practice for many diseases. Thoughtfully designed and well-performed prospective minimal residual disease (MRD)-based monitoring studies were first implemented in childhood acute lymphoblastic leukaemia (ALL) therapy protocols in the 1990s. We learnt that the presence of MRD above a certain threshold (which specifically depends on the disease or disease subtype, the given chemotherapy and the time point of measurement) predicts relapse [2]. Today, prospective risk adaptation of chemotherapy, indications for stem-cell transplantation (SCT) and selection of a particular stem-cell donor (matched versus unmatched, sibling versus unrelated, haplo-identical, etc.) are all critically influenced by MRD assessment, which can now be considered as a routine diagnostic procedure, at least in childhood ALL and many other haematological tumours. During and after chemotherapy, the surviving leukaemic cells are mostly traced by PCR amplification of either clonally expanded antigen (immunoglobulin Ig or $\mathrm{T}$ cell) receptor rearrangements or leukaemia-specific genetic aberrations, e.g. fusion genes generated by chromosomal translocations [3]. In children with B-precursor ALL, the TEL/AML1, E2A/Pbx1 and various MLL-fusion genes are the most commonly found hybrid variants. For some of them (e.g. the MLL/ ENL fusion), transgenic or serial transplantation in vivo

\footnotetext{
A. Borkhardt $(\triangle)$

Clinic of Pediatric Oncology, Haematology and Clinical

Immunology, Centre for Child and Adolescent Health,

Heinrich-Heine University,

40225 Düsseldorf, Germany

e-mail: lesch@med.uni-duesseldorf.de
}

models exist which convincingly demonstrate the powerful transforming capabilities of the fusion gene alone once it becomes expressed in a susceptible cell background [4-6]. Expression of the weaker TEL/AML1 oncoprotein, in contrast, is not leukaemogenic by itself and thus needs additional co-operating genetic aberrations for overt clinical leukaemia [7]. In any case, these particular gene fusions characterise specific risk groups and are often associated with additional immunological or clinical findings [8]. Together with antigen-receptor rearrangements, they have been widely used for MRD studies in the past. However, the study by Shalapour et al. [9] in this issue nicely demonstrates that these individual clone- or leukaemiaspecific molecular markers are now detected not only in the leukaemic blast cells themselves but also in the surrounding mesenchymal stem cell (MSC) fraction, albeit in lower frequency. Of note, the fraction of translocation-positive MSCs varied among the patients tested from $10 \%$ to $54 \%$, and one patient remained MLL-positive in his host MSC fraction, even after double myeloablative SCT. From the methodological point of view, the MSC characterisation as presented in that study [9] is outstanding in its formidable number of control experiments, virtually excluding the possibility of a simple contamination by residual leukaemic cells. Among other techniques, the authors combined FISH technique with immunocytochemistry to really document the presence of the leukaemia-associated fusion genes in the MSC fraction.

This very elegant study also nicely illustrates how prosperous collaborative projects between clinical and basic researchers can be conducted. The work challenges our current view and paves the way for further experiments. Most importantly, it suggests a clonal relationship between MSC and leukaemic cells. The expression of the CD133 marker may be helpful in characterising the "stemness" of 
an assumed common progenitor cell. The leukaemiaassociated genetic aberrations remained stable in the MSC fraction even when the children were virtually free of disease. Thus, neither chemotherapy alone nor even chemotherapy followed by SCT was able to eradicate stromal mesenchymal cells in the children's bone marrow. Because only three out of eight patients showed clonespecific Ig sequences in addition to their leukaemia-specific gene fusion, however, one may predict significant differences in MRD quantification depending on whether Ig or chromosomal fusion genes are used for calculation of residual tumour burden. But no clean comparative experimental MRD study has been published yet that addressed this clinically most relevant question. One pitfall in such a comparative PCR-based MRD study should carefully be considered beforehand. Fusion gene transcripts associated with chromosomal translocations are mostly quantified by RT-PCR (with only a few exceptions) whereas the more robust DNA PCR can be used for amplification of clonal Ig or TCR rearrangements. Although there are obvious methodological differences in terms of sensitivity and stability between DNA- and RNA-based quantification procedures, the number of leukaemia-derived transcripts most likely does not linearly correspond to the number of residual leukaemic cells.

Given the relatively high frequency of translocationpositive MSCs in remission, up to $54 \%$ in the study of Shalapour et al. [9], one might think of serial conventional karyotyping even in children in long-term remission. However, such a study would likely not get ethical approval because "routine" bone marrow puncture after finishing a quite complex chemotherapy protocol lasting 2 years is clearly not justified. On the other hand, and as stated by the authors, examination of persisting chromosomal aberrations in MSCs - either numerical or structural ones-may be worthwhile for understanding the development of secondary bone tumours. It is very likely and supported by the growing experimental evidence that MSCs - or derivatives thereof-are natural progenitors for Ewing Sarcomas and Osteosarcomas, both of which are frequently found secondary neoplasms after ALL therapy. Like all good work, the paper opens numerous new questions. The generation of MSCs was technically successful in 49 out of 60 patients. What causes this inherent bias, or does it have some biological significance? Do some chromosomal aberrations prevent or facilitate the establishment of MSC cultures more than others do? Do karyotype anomalies persist also in MSCs from children with an easy-to-cure, non-relapsed leukaemia?

Although another group recently reported that MLL/AF4 fusion transcripts are expressed in 4-8\% of MSCs isolated from four children with this particular genetic leukaemia subtype [10], some remarkable differences between these two studies catch our attention. Menendez et al. [10] failed to detect other chimeric mRNAs like BCR/ABL, AML1/ ETO, MLL/AF9, MLL/AF10, TEL/AML1 and MLL/ENL. The latter two fusions were clearly demonstrated by Shalapour et al. Both fusions arise during foetal development in utero, possibly in a mesodermal pre-haematopoietic precursor population. Remarkably, monoclonal Ig gene rearrangements could not be seen in any MSC sample by the Spanish group, whereas Shalapour et al. demonstrated their presence in three out of eight samples. The amplification plots of Menendez et al. (see Fig. $2 b$ in their paper) suggest that the MLL/AF4 expression in MSCs is two or three orders of magnitude lower than in corresponding

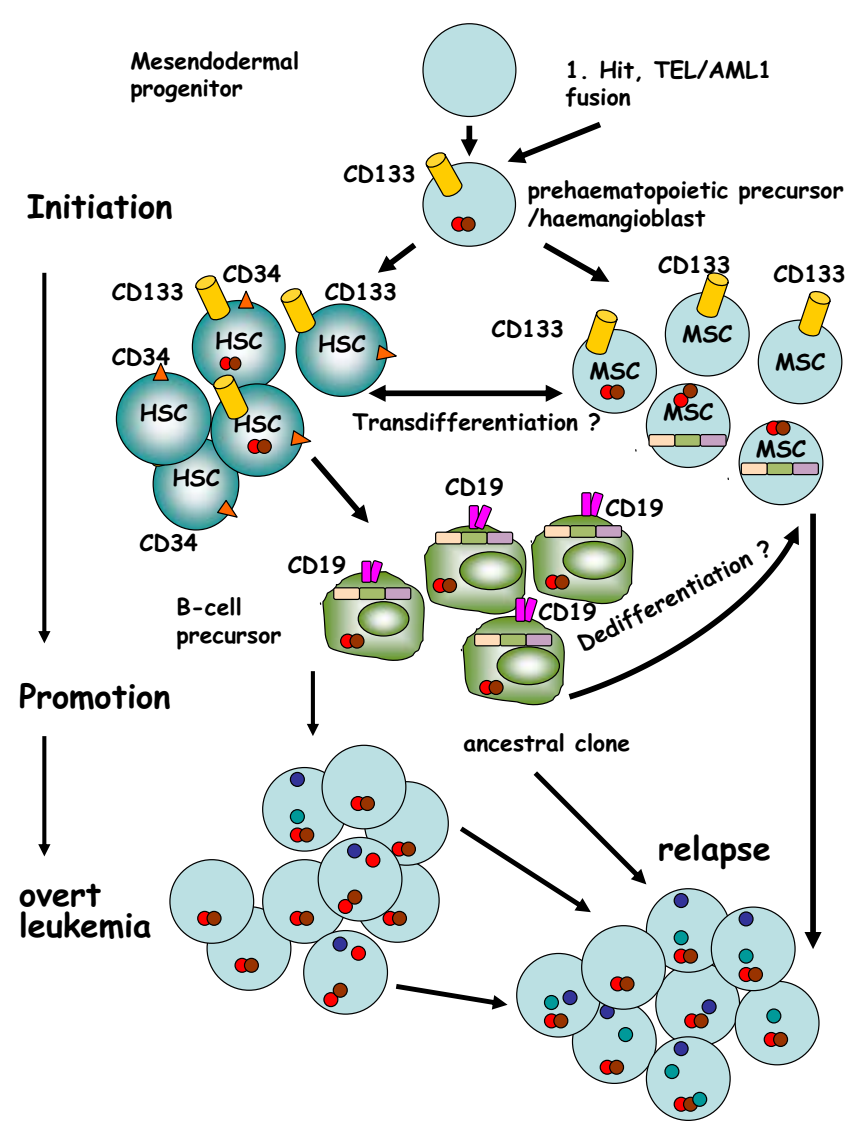

Fig. 1 Hypothetical model of various cellular origins of leukaemia relapses. The first genetic leukemogenic hit, here exemplified by the TEL/AML1 rearrangement, occurs in pre-haematopoietic precursor that may further differentiate along the hematopoietic (HSC) and the mesenchymal stem cells (MSC) lineage. Trans-differentiation or even dedifferentiation from a more committed B-precursor cell may contribute to a dynamic pool of MSCs in which some cells express the TEL/AML1 fusion and even immunoglobulin rearrangements. Ancestral pre-leukaemic clones acquire further genetic hits leading to outgrowth of mixed, heterogeneous leukemic cells at diagnosis. The chemotherapy surviving relapse clone may thus derive from various cellular origins, e.g. an ancestral pre-leukaemic clone, the predominant diagnostic clone, a minor diagnostic subclone or even from the MSC fraction. The Ig rearrangements are depicted as boxes in MSCs or B-precursor cells, respectively 
leukaemic blast cells. The typical sigmoidal shape of the amplification curve - associated with effective product accumulation during the exponential phase of PCR-is lacking; furthermore, the exact relative expression of MLL/ AF4 compared to the housekeeping ABL mRNA control is not given. Thus, to date, it is not entirely clear to me at which level the fusion mRNA is indeed expressed in MSCs. The most convincing set of data, in my view, is the expression of the translated $\mathrm{E} 2 \mathrm{~A} / \mathrm{Pbx} 1$ chimeric oncoprotein in long-term culture of SV40-Tag transduced MSCs which were derived from a patient with ALL and translocation $t(1 ; 19)$. Lymphoblastic leukaemia cells rapidly die in culture, strongly arguing against a contamination of the MSCs culture.

Besides all these practical questions, future basic studies should address the specific role of MSCs in leukaemia development. How is the tumour-stroma relationship defined at its molecular level? Is the genetic heterogeneity of primary MSCs directly related to the evolution of various genetic subcomponents of ALL cells? Genome-wide genetic analysis of paired diagnostic/relapse ALL samples recently showed that in more than half of all the relapsed cases, the cells acquired additional genetic lesions from an ancestral (pre-leukemic) but not directly from the bulky clone present at primary diagnoses [11]. The relapse clone exists in most cases only as a minor subclone at diagnosis.

Having the documented plasticity of stem or progenitor cells in mind, one might even speculate that there is an ongoing interchange between "haematopoietic/leukaemia lineage-progenitor cells" and "stroma lineage progenitor cells". Perhaps a genetically heterogeneous mesodermal precursor population feeds the cancer stem cell (CSC) pool directly via MSCs? This would imply that multiple cancer stem cells exist and that these cells may vary in phenotype and genotype. As Mel Greaves put it: CSC are a "moving target" (Fig. 1) [12].

Finally, I would like to point to another aspect that might be relevant to the topic: "Healthy" or "normal" MSCs usually suppress T-cell effector function and promote development of immunosuppressive regulatory $\mathrm{T}$ cells (Tregs) by a variety of mechanisms, e.g. indoleamine 2,3dioxygenase-mediated Tryptophan depletion [13]. In addition, MSCs can modulate NK- and B-cell function, antigen presentation by dendritic cells (DC) and monocytes [14]. These multi-faceted, but not fully-defined properties of MSCs, encouraged many clinicians to implement MSCs in approaches for treatment of multiple sclerosis, diabetes mellitus type 1, graft-versus-host disease, graft failure after SCT, inflammatory bowel disease or osteogenesis imperfecta [15].

With respect to leukaemia development, genetically aberrant MSCs may interfere with normal, physiological immunesurveillance, e.g. by augmenting suppression of
T-cell effector function or inhibiting DC maturation and proliferation. For prevention of overt clinical leukaemia, the effective, continuous autologous T-cell response against immunogenic B-precursor blast cells may be crucial especially in situations with low leukaemic (pre-leukemic) cell burden. Of note, data from the Greaves' group suggest that $1 \%$ of all healthy newborns harbour a TEL/AML1 positive clone in their cord blood at birth [16]. In $99 \%$ of those cases, however, the TEL/AML1 clone disappeared or does at least not rise to clinically overt leukaemia during later life. Perhaps the TEL/AML1+ cells are effectively cleared by cytotoxic responses of their autologous $\mathrm{T}$ or NK cells.

In such a scenario, MSCs with normal or aberrant karyotype may significantly differ in their functional properties to modulate their own immune cells. At a first glance, ectopic expression of the fusion genes MLL/AF4, MLL/AF9 and TEL/AML1 does not seem to alter their proliferation, cell-cycle distribution, apoptosis rate or time point of senescence [10]. However, to the best of my knowledge, there are no functional data comparing the immunomodulatory capacity of MSCs with and without leukaemia-specific fusion genes expressed.

The leukaemia-stroma relationship seems to be far more complex than previously thought. In any case, the cure rate in childhood ALL nowadays suggests that we are able to hit the "moving target" of CSC in the great majority but not in all of our patients. In terms of understanding the striking differences in outcome, Shalapour et al. [9] have now introduced a new player in the puzzling game. It might well be that our current belief about the origin of relapses in childhood ALL awaits further correction soon.

\section{References}

1. Lee MS, Chang K-S, Cabanillas F, Freireich EJ, Trujillo J, Stass SA (1987) Detection of minimal residual cells carrying the $t$ $(14 ; 18)$ by DNA sequence amplification. Science 237:175-178

2. van Dongen JJ, Seriu T, Panzer-Grümayer ER, Biondi A, PongersWillemse MJ, Corral L, Stolz F, Schrappe M, Masera G, Kamps WA, Gadner H, van Wering ER, Ludwig WD, Basso G, De Bruijn MA, Cazzaniga G, Hettinger A, van der Does-van den Berg A, Hop WC, Riehm H, Bartram CR (1998) Prognostic value of minimal residual disease in acute lymphoblastic leukaemia in childhood. Lancet 352(9142):1731-1738

3. Bruggemann M, Schrauder A, Raff T, Pfeifer H, Dworzak M, Ottmann OG, Asnafi V, Baruchel A, Bassan R, Benoit Y, Biondi A, Cave H, Dombret H, Fielding AK, Foa R, Gokbuget N, Goldstone AH, Goulden N, Henze G, Hoelzer D, JankaSchaub GE, Macintyre EA, Pieters R, Rambaldi A, Ribera JM, Schmiegelow K, Spinelli O, Stary J, von SA, Kneba M, Schrappe M, van Dongen JJ (2009) Standardized MRD quantification in European ALL trials. Proceedings of the Second International Symposium on MRD assessment in Kiel, Germany, 18-20 September 2008. Leukemia 
4. Hess JL, Bittner CB, Zeisig DT, Bach C, Fuchs U, Borkhardt A, Frampton J, Slany RK (2006) c-Myb is an essential downstream target for homeobox-mediated transformation of hematopoietic cells. Blood 108(1):297-304

5. Mueller D, Bach C, Zeisig D, Garcia-Cuellar MP, Monroe S, Sreekumar A, Zhou R, Nesvizhskii A, Chinnaiyan A, Hess JL, Slany RK (2007) A role for the MLL fusion partner ENL in transcriptional elongation and chromatin modification. Blood 110 (13):4445-4454

6. Schwieger M, Schuler A, Forster M, Engelmann A, Arnold MA, Delwel R, Valk PJ, Lohler J, Slany RK, Olson EN, Stocking C (2009) Homing and invasiveness of MLL/ENL leukemic cells is regulated by MEF2C. Blood 114(12):2476-2488

7. Fischer M, Schwieger M, Horn S, Niebuhr B, Ford A, Roscher S, Bergholz U, Greaves M, Lohler J, Stocking C (2005) Defining the oncogenic function of the TEL/AML1 (ETV6/RUNX1) fusion protein in a mouse model. Oncogene 24(51):7579-7591

8. Pui CH, Schrappe M, Ribeiro RC, Niemeyer CM (2004) Childhood and adolescent lymphoid and myeloid leukemia. Hematology Am Soc Hematol Educ Program 2004:118-145

9. Shalapour S, Eckert C, Seeger K, Pfau M, Prada J, Henze G, Blankenstein T, Kammertoens T (2010) Leukemia-associated genetic aberrations in mesenchymal stem cells of children with acute lymphoblastic leukemia. J Mol Med, in press
10. Menendez P, Catalina P, Rodriguez R, Melen GJ, Bueno C, Arriero M, Garcia-Sanchez F, Lassaletta A, Garcia-Sanz R, Garcia-Castro J (2009) Bone marrow mesenchymal stem cells from infants with MLL-AF4+ acute leukemia harbor and express the MLL-AF4 fusion gene. J Exp Med 206(13):3131-3141

11. Mullighan CG, Phillips LA, Su X, Ma J, Miller CB, Shurtleff SA, Downing JR (2008) Genomic analysis of the clonal origins of relapsed acute lymphoblastic leukemia. Science 322(5906):13771380

12. Greaves M (2009) Darwin and evolutionary tales in leukemia. Hematology Am Soc Hematol Educ Program 2009:3-12

13. Meisel R, Zibert A, Laryea M, Gobel U, Daubener W, Dilloo D (2004) Human bone marrow stromal cells inhibit allogeneic T-cell responses by indoleamine 2, 3-dioxygenase-mediated tryptophan degradation. Blood 103(12):4619-4621

14. Nauta AJ, Fibbe WE (2007) Immunomodulatory properties of mesenchymal stromal cells. Blood 110(10):3499-3506

15. Kode JA, Mukherjee S, Joglekar MV, Hardikar AA (2009) Mesenchymal stem cells: immunobiology and role in immunomodulation and tissue regeneration. Cytotherapy 11(4):377-391

16. Mori H, Colman SM, Xiao Z, Ford AM, Healy LE, Donaldson C, Hows JM, Navarrete C, Greaves M (2002) Chromosome translocations and covert leukemic clones are generated during normal fetal development. Proc Natl Acad Sci U S A 99(12):8242-8247 